\title{
Les frontières de la métropolisation
}

Inégalités sociospatiales d'accès a l'eau et indicateurs de pauvreté à La Paz, Bolivie

The Frontiers of Metropolization. Sociospatial Inequalities in the Access to Water and Poverty Indicators in La Paz, Bolivia

\section{Franck Poupeau}

\section{CpenEdition}

\section{Journals}

Édition électronique

URL : http://journals.openedition.org/transcontinentales/368

DOI : 10.4000/transcontinentales.368

ISBN : 978-2-7351-1559-4

ISSN : 1775-397X

Éditeur

Editions de la maison des sciences de l'homme

\section{Édition imprimée}

Date de publication : 30 juin 2009

Pagination : 81-104

ISBN : 978-2-200-92512-3

ISSN : 1950-1684

\section{Référence électronique}

Franck Poupeau, «Les frontières de la métropolisation », Transcontinentales [En ligne], 7 | 2009, document 5, mis en ligne le 24 mars 2011, consulté le 07 septembre 2020. URL : http:// journals.openedition.org/transcontinentales/368; DOI : https://doi.org/10.4000/transcontinentales. 368 


\title{
Les frontières de la métropolisation
}

\author{
Inégalités sociospatiales d'accès a l'eau \\ et indicateurs de pauvreté à La Paz, Bolivie
}

Franck Poupeau

Si une des grandes orientations de la sociologie urbaine consiste à comprendre les effets de la mondialisation sur les métropoles des pays du Nord - les global cities -, l'étude des transformations des villes du Sud apparaît comme le domaine réservé de la géographie et de l'ethnographie, qui s'attachent depuis une trentaine d'années aux formes de ségrégation urbaine liées à la croissance des quartiers périphériques. L'assimilation implicite entre pays du Nord et métropoles modernes est sans doute pour beaucoup dans le désintérêt de la sociologie envers les villes de pays encore désignés sous l'appellation de «Tiers Monde» - et donc relevant seulement d'études en développement. Favelas de Rio de Janeiro, ranchos de Caracas, villas miserias de Buenos Aires, barriadas de Lima, etc. - pour ne citer que l'Amérique latine : ces quartiers périphériques en expansion, qui représentent «le mode d'urbanisation informel, précaire ou illégal de la grande majorité des citadins dans le monde ${ }^{1}$ ", offrent pourtant un condensé de problèmes relevant traditionnellement de la sociologie. Ségrégation sociale, spatiale et raciale affectant des populations qui n'ont bien souvent d'autres ressources que des emplois informels et sous-payés, manque d'infrastructures sanitaires et éducatives, développement d'une violence structurelle et incontrôlée, etc. : cette "marginalité urbaine» ne peut être séparée de la croissance démographique des villes du Sud. L'Amérique latine a en particulier connu une "explosion urbaine» plus précoce que les autres continents ${ }^{2}$ : enclenchée dans les années 1920 avec d'amples migrations rurales, cette "explosion" s'est recomposée au cours des années 1970 avec le poids démographique des villes elles-mêmes, au point de produire des taux d'urbanisation similaires à ceux des pays du Nord. Ces villes et leurs périphéries en expansion constituent

1 - M. Agier, L'invention de la ville: banlieues, townships, invasions et favelas, Amsterdam, Overseas Publishers Association et Paris, Éditions des archives contemporaines, 1999, p. 7.

2 - J.-F. Troin, Les métropoles des Sud, Paris, Ellipses, 2000, p. 5.

Franck Poupeau: Pensionnaire de l'Institut français d'études andines IFEA (UMIFRE 17 CNRSministère des Affaires étrangères et européennes). 
ainsi une sorte de laboratoire in situ des processus de transformation des espaces urbains, généralement désignés par la notion de métropolisation.

Puisqu'une métropole regroupe activités productives, contrôle des échanges, fonctions de régulation et de domination politique, la métropolisation est censée désigner un accroissement du pouvoir de commandement d'une grande ville sur un territoire élargi, une concentration des activités économiques et sociales et une ouverture aux flux mondiaux de commerce et de communication ${ }^{3}$. Généralement perçu dans son lien à l'économie nationale ou globalisée, le processus de métropolisation est pourtant aussi associé à la production de formes de ségrégation sociospatiale générant des tensions particulièrement visibles sur les zones frontalières entre les communes. Depuis le milieu des années 1990, la ville de La Paz, siège du gouvernement de l'État plurinational de Bolivie, constitue un cas exemplaire des problèmes suscités par une croissance urbaine exponentielle: conflits entre administrations municipales, la plupart du temps pour des questions de limites territoriales entre La Paz et les mairies voisines qui lui reprochent sa politique "hégémonique» d'un point de vue territorial comme économique; conflits entre résidents de ces zones frontalières, partagés entre la volonté de rejoindre la municipalité «centrale» de La Paz pour bénéficier de ses services urbains, et celle de rester dans les communes, plus rurales, moins bien équipées mais aussi bien moins coûteuses en termes d'impôts locaux et de taxes foncières. Ces tensions sociales sont d'autant plus vives que les municipalités apparaissent comme inégales : la ville "centrale» concentre toutes les formes de capitaux, économiques, culturels et sociaux, qu'elle peut mobiliser pour ses projets d'aménagement territorial et d'extension géographique. Engoncée dans une vallée à 3600 mètres au pied des montagnes de la Cordillère royale, la ville de La Paz, dont la population s'élève à 850000 personnes à la fin des années 2000, cherche à contourner les limitations spatiales de son emplacement : les pics rocheux qui l'entourent, les ravines instables qui séparent les quartiers, constituent autant de facteurs qui entravent désormais son extension et multiplient la spéculation immobilière ou les implantations sauvages sur les rares zones planes encore inhabitées. Surplombée par la ville de El Alto, l'excroissance urbaine qui l'a désormais dépassée en nombre d'habitants (900 000 au seuil de 2010) et qui se développe sur l'altiplano bolivien, La Paz n'a d'autre solution que de se tourner vers les autres municipalités, qu'elles soient rurales (Palca, Achocalla) ou résidentielles (Mallasa, Rio Seco).

Lorsque dans son plan de développement urbain de $2007^{4}$, la mairie de La Paz met l'accent sur les transformations locales et les projets à développer, c'est la métropolisation qui est invoquée comme base d'un "développement harmonieux" entre la ville et les autres «communes sœurs» de l'aire métropolitaine, afin «de rétablir un équilibre géopolitique national, de redynamiser le développement économique et productif du département de La Paz et d'établir un pôle de gravitation sur le Pacifique». Le corollaire de cette ouverture nationale et internationale réside dans

3 - C. Lacour et S. Puissant (dir.), La métropolisation. Croissance, diversité, fractures, Paris, Anthropos, 1999.

4 - Primera Asamblea del Municipio, Declaración final, La Paz, 31 octobre 2008. 
le soutien aux "travaux stratégiques" de transformation du territoire municipal, qu'il s'agisse de faciliter les transports, d'améliorer le cadre de vie ou de renforcer les entreprises municipales d'eau et de gaz. L'espace urbain de La Paz est ainsi parsemé de chantiers, ici pour rénover le réseau de distribution des eaux, là pour tenter de délimiter un espace vert, pris en tenailles entre un nouvel échangeur routier et un quartier en construction, là encore pour ouvrir une nouvelle route vers les communes limitrophes. Ce sont justement les zones frontalières avec les municipalités voisines de l'agglomération centrale qui concentrent les indicateurs de sous-équipement en services urbains, de moindre accès aux soins et à l'éducation, d'habitat le plus sommaire et d'activité économique la plus précaire ${ }^{5}$. Ces zones périphériques, dans leurs difficultés et leurs dysfonctionnements, sont donc les plus susceptibles de révéler les logiques à l'œuvre dans le processus de métropolisation. La problématique développée ici repose sur l'idée que les inégalités d'accès au service de distribution d'eau et d'assainissement constituent "un indicateur des rapports d'inégalité sociale ${ }^{6} "$, en raison des coûts d'équipement élevés, de la médiocre qualité du service dans les zones les plus pauvres et des risques environnementaux: les difficultés d'accès au réseau des quartiers périphériques en expansion de l'agglomération permettent alors de cerner non seulement les conflits générés par le processus métropolisation en cours, mais aussi de revenir sur les indicateurs de pauvreté utilisés par les politiques d'aménagement urbain. Elles permettent de repérer la cohérence des styles de vie associés au fait de vivre dans tel ou tel quartier, à la jonction des dimensions sociales et spatiales.

\section{Une enquête sur les inégalités sociospatiales d'accès à l'eau}

Ce sont les problèmes d'accès à l'eau potable et à l'assainissement qui rendent particulièrement sensibles les relations entre les villes de l'espace métropolitain. L'aire de concession de l'entreprise municipale est en effet limitée aux villes de La Paz et de El Alto : la participation financière est jugée trop élevée par les administrations des villes voisines pour rendre l'extension du réseau possible. L'entreprise municipale est par ailleurs trop récente pour pouvoir assumer des investissements importants en dehors du réseau existant : elle n'a été créée qu'en 20077. De 1997 à 2007, la distribution de l'eau avait en effet été assurée par le consortium Aguas del Illimani, dont l'actionnaire principal était l'entreprise française Suez-Lyonnaise des eaux. Cette privatisation du service avait certes permis un transfert de technologies d'exploitation et une «modernisation» des structures de l'entreprise municipale préexistante $e^{8}$; mais la délimitation trop stricte de l'aire de distribution, qui n'incluait pas les quartiers périphériques en expansion, avait fini

5 - Gobierno Municipal de La Paz (GMLP), Atlas del municipio de La Paz. Una lectura sociodemográfica desde las Organizaciones Territoriales de Base, La Paz, Consejo de población para el desarollo sostenible (CODEPO) - GMLP - Institut de recherche pour le développement (IRD), 2006.

6 - G. Meublat, "La rénovation des politiques de l'eau dans les pays du Sud», Revue Tiers Monde, t. XLII, $\mathrm{n}^{\circ} 166,2001$, p. 249-258.

7 - F. Poupeau, «Les ambivalences de la participation communautaire. Le cas du service de distribution des eaux à El Alto, Bolivie», Autrepart, n 47,2008 , p. 245-254.

8 - K. Komives, "Designing pro-poor water and sewer concessions : early lessons from Bolivia», Water Policy, 2001 (3), p. 61-79. 
par susciter le mécontentement des organisations sociales. La ville de El Alto, dont le taux de croissance démographique annuel oscille encore entre 5 et $9 \%$ depuis la fin des années 1990, s'était particulièrement mobilisée ${ }^{9}$, alors que La Paz possédait, quant à elle, un taux de couverture suffisamment important (plus de $95 \%$ ) pour ne pas susciter de protestations. Mais à partir de l'élection d'Evo Morales à la présidence de la République en décembre 2005, le contexte de renationalisation des entreprises d'exploitation des ressources naturelles (en particulier le gaz) et de communication avait provoqué le départ, après plusieurs mois de négociations, d'Aguas del Illimani. La remunicipalisation de janvier 2007 n'a cependant pas entraîné de changements substantiels en termes d'organisation du service: les critères requis pour équiper les nouveaux quartiers se sont assouplis, mais pas de façon suffisamment nette pour permettre l'extension du réseau aux quelques zones en expansion de La Paz, frontalières avec les municipalités voisines ${ }^{10}$.

C'est dans ces quartiers que l'enquête a été menée entre avril et août $2008^{11}$, soit un peu plus d'un an après la remunicipalisation du service d'eau. Elle a consisté à distribuer des questionnaires selon une double logique d'échantillonnage: un premier échantillon "territorial» a consisté à choisir, à la frontière des municipalités de La Paz et Palca, là où les tensions sont les plus vives, des quartiers représentatifs de la diversité de situations possibles, en termes d'accès à l'eau, de caractéristiques matérielles de l'habitat, de situation géographique, de propriétés socioéconomiques de la population et de distance au centre-ville de La Paz (voir l'encadré "Plan et caractéristiques des quartiers étudiés»); ce premier échantillon territorial étant défini, il a été décidé de recueillir dans chacun de ces quartiers un nombre de questionnaires équivalent à 10\% des habitations. L'unité de base est en effet le logement familial (vivienda), qui constitue la donnée essentielle du recensement national de 2001, dont le questionnaire diffusé a repris certaines questions afin d'établir une base de données comparable sur des quartiers en expansion qui n'existaient pas sous cette forme à l'époque. Cent cinquante-six questionnaires ont ainsi été recueillis, et exploités statistiquement à l'aide du logiciel SPSS. Si les quartiers étudiés ont été choisis pour leur représentativité par rapport à la diversité des situations possibles à la périphérie de La Paz, ils n'en possèdent pas moins certaines caractéristiques communes, qui permettent de définir un profil global du terrain d'investigation : une installation relativement ancienne et stable, des chefs de famille exerçant pour leur grande majorité des métiers manuels et peu payés, un ancrage rural plus prononcé que dans d'autres parties de la ville, un habitat souvent sommaire et déficient en termes de services urbains. Seuls deux quartiers périphériques, Chijipata et les condominiums de Cota Cota, se révèlent plus favorisés. Les résidents des quartiers étudiés présentent des caractéristiques qui, sans relever complètement de situations d'extrême pauvreté, les situent (suite p. 89)

9 - F. Poupeau, «El Alto : una ficción política», conférence prononcée à l'Université de tous les savoirs, des Andes à l'Amazonie, le 21 septembre 2009 à La Paz, Bolivie.

10 - F. Poupeau, «Les ambivalences de la participation communautaire...», art. cit.

11 - Cette enquête a été menée avec l'aide et la complicité amicale de deux stagiaires de l'Institut d'études politiques de Rennes, option Gestion des services urbains: Antoine Brochet et Florian Marchadour. 


\section{Plan et CARActéristiQues des quartiers Étudiés}

Liste des quartiers :
1 - Mirador Turístico
2 - Apaña
3 - Alto Ovejuyo
4 - Condominiums
5 - Chijipata
(regroupé avec
le précédent dans
I'analyse statistique)
6- Chicani
7 - Pokechaka
8 - 24 de Junio

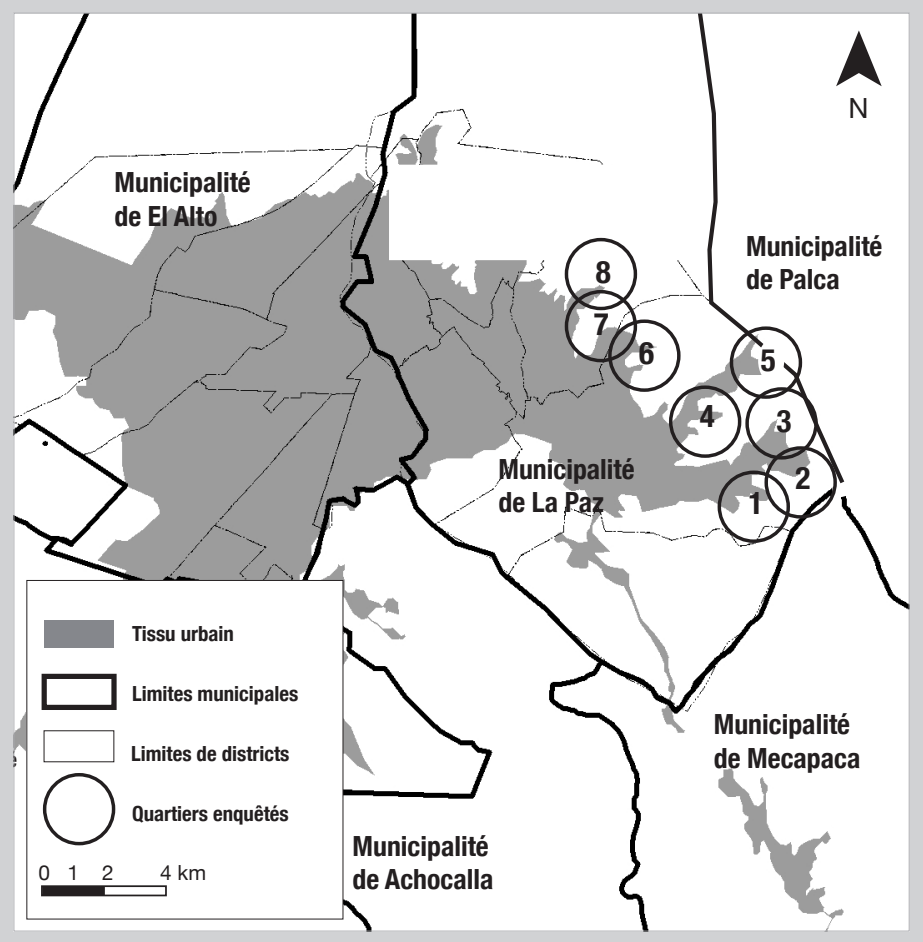

\begin{tabular}{|c|c|c|c|c|c|}
\hline Quartier & $\begin{array}{l}\text { Environnement } \\
\text { physique }\end{array}$ & $\begin{array}{l}\text { Accessibilité } \\
\text { et transports }\end{array}$ & $\begin{array}{l}\text { Connexion } \\
\text { au réseau d'eau }\end{array}$ & Type d'habitat & $\begin{array}{l}\text { Population } \\
\text { active }\end{array}$ \\
\hline $\begin{array}{l}\text { Mirador } \\
\text { turístico }\end{array}$ & Flan de montagne & $\begin{array}{l}\text { Proximité } \\
\text { arrêts de bus }\end{array}$ & $\begin{array}{l}\text { Problèmes } \\
\text { de pression }\end{array}$ & $\begin{array}{l}\text { Maisons } \\
\text { en briques }\end{array}$ & $\begin{array}{l}\text { Employés, } \\
\text { retraités }\end{array}$ \\
\hline Apaña & Vallée urbanisée & Bus réguliers & Aucune & Briques et adobe & $\begin{array}{l}\text { Ouvriers, } \\
\text { agriculteurs }\end{array}$ \\
\hline $\begin{array}{r}\text { Alto } \\
\text { Ovejuyo }\end{array}$ & Flan de montagne & $\begin{array}{l}\text { Loin } \\
\text { des arrêts de bus }\end{array}$ & $\begin{array}{l}\text { Problèmes } \\
\text { de pression }\end{array}$ & $\begin{array}{l}\text { Principalement } \\
\text { adobe }\end{array}$ & $\begin{array}{l}\text { Ouvriers, } \\
\text { maçons }\end{array}$ \\
\hline $\begin{array}{r}\text { Condomi- } \\
\text { niums }\end{array}$ & $\begin{array}{l}\text { Plateau dégagé } \\
\text { zone sud }\end{array}$ & Véhicules individuels & $\begin{array}{l}\text { Connexion } \\
\text { commune }\end{array}$ & $\begin{array}{l}\text { Maisons de } \\
\text { briques/pierre }\end{array}$ & Cadres \\
\hline Chijipata & $\begin{array}{l}\text { Vallée urbanisée à } \\
\text { faible densité }\end{array}$ & $\begin{array}{l}\text { Bus et véhicules } \\
\text { individuels }\end{array}$ & $\begin{array}{l}\text { Connexions } \\
\text { individuelles }\end{array}$ & $\begin{array}{l}\text { Maisons de } \\
\text { briques/pierre }\end{array}$ & $\begin{array}{l}\text { Employés } \\
\text { et cadres }\end{array}$ \\
\hline Chicani & $\begin{array}{l}\text { Vallée et cultures à } \\
\text { flan de montagne }\end{array}$ & $\begin{array}{l}\text { Bus réguliers } \\
\text { Voitures individuelles }\end{array}$ & $\begin{array}{l}\text { Coopératives } \\
\text { gérant les sources } \\
\text { locales }\end{array}$ & $\begin{array}{l}\text { Habitat mixte } \\
\text { Briques }\end{array}$ & $\begin{array}{l}\text { Agriculteurs } \\
\text { et } \\
\text { indépendants }\end{array}$ \\
\hline Pokechaka & Flan de montagne & $\begin{array}{l}\text { Loin } \\
\text { des arrêts de bus }\end{array}$ & Bornes fontaines & Briques et adobe & $\begin{array}{l}\text { Ouvriers, } \\
\text { employés }\end{array}$ \\
\hline $\begin{array}{l}24 \text { de } \\
\text { Junio }\end{array}$ & Flan de montagne & $\begin{array}{l}\text { Loin } \\
\text { des arrêts de bus }\end{array}$ & Bornes fontaines & Briques et adobe & $\begin{array}{l}\text { Ouvriers, } \\
\text { employés }\end{array}$ \\
\hline
\end{tabular}


Ces quartiers se situent tous à divers endroits de la frontière entre la municipalité de La Paz et celle de Palca, une municipalité essentiellement rurale comportant 14000 habitants, répartis sur un territoire de $743 \mathrm{~km}^{2}$ dans des petits villages ou de petites bourgades; les deux tiers de la population active sont agriculteurs. En 2008, lors d'une réunion de conciliation organisée par la préfecture du département, la municipalité de Palca a revendiqué les zones suivantes: Apaña, Ovejuyo, Achumani, Irpavi, Chicani, Pampahasi, Chuquiaguillo (dans laquelle se trouvent 24 de Junio et Pokechaka), Calacoto Alto (dans laquelle se trouvent les condominiums), Chinchaya (dans laquelle se trouve Chijipata), ainsi qu'une quinzaine d'autres quartiers.

Presque la moitié (49\%) des habitants a emménagé depuis plus de quinze ans; une partie conséquente des habitants a toujours vécu dans certains quartiers comme Chicani ; $21 \%$ se sont installés entre 1994 et 2001, tandis que $30 \%$ se sont installés depuis 2001 , I'augmentation étant due à la pression démographique croissante sur les zones périphériques. La presque totalité des habitants interrogés (95\%) vit dans une maison particulière, avec $91 \%$ de propriétaires (pour $5 \%$ de locataires et $4 \%$ de maisons prêtées). Seulement $70 \%$ d'entre eux possèdent un titre de propriété, et $20 \%$ une autre maison en dehors du quartier. L'enquête révèle que $42 \%$ des chefs de famille sont nés à La Paz, la grande majorité des autres venant d'autres provinces du département.

En ce qui concerne les logements, $42 \%$ d'entre eux sont en adobe (un mélange de terre et de pierres), ce qui correspond à la moyenne de I'agglomération de La Paz (selon le recensement de 2001), et $33 \%$ seulement sont équipés de toilettes, dont la moitié seulement débouche sur une fosse d'aisance (pozo ciego) et un tiers sur un réseau d'assainissement (les deux tiers des habitants font leurs besoins à l'extérieur) : le sous-équipement est net par rapport à l'agglomération de La Paz (84\%) mais inférieur à celui de Palca (22\%). Si l'équipement électrique semble relativement satisfaisant (90\% des habitations), l'accès au réseau d'eau à l'intérieur de la maison ne concerne que $16 \%$ des logements (cañería en red a dentro de la vivienda), et $11 \%$ à l'intérieur du lot (cañería en red a dentro del lote). Une majorité (55\%) des habitants des quartiers enquêtés se procure donc de l'eau dans des puits situés à proximité de leur logement, et $16 \%$ bénéficient d'un système de coopérative pour distribuer ensuite l'eau disponible; en revanche, moins de $2 \%$ des habitants ont recours à des camions-citernes, contrairement à la situation dans d'autres parties de I'agglomération (El Alto, Achocalla). Dans le domaine de l'hygiène et de la santé, la gestion des eaux usées et des déchets laisse apparaître les insuffisances du service municipal : alors que $92 \%$ des foyers interrogés se débarrassent des eaux usées en les jetant dehors, $80 \%$ mettent leurs déchets dans un endroit prévu à cet effet, en ayant connaissance de l'existence d'un service de collecte des ordures, relativement présent sur tout le territoire de La Paz. De ce point de vue, les quartiers périphériques révèlent une situation de sous-équipement structurel par rapport à l'ensemble de l'agglomération, où I'accès à l'eau potable et à l'assainissement concerne respectivement $91 \%$ et $84 \%$ de la population. À Palca, la population n'est équipée qu'à $47 \%$ et $22 \%$.

Les informations recueillies sur le type d'eau utilisée pour les usages quotidiens permettent de préciser certaines zones d'ombre du recensement national de 2001, dont les questions sur les conditions de logement ont été utilisées ci-dessus. En effet, certaines connexions à domicile ne sont pas le fait de I'entreprise municipale EPSAS : elles sont réalisées par une coopérative locale ou un système géré collectivement, en particulier 
dans les quartiers de Chicani, Apaña ou 24 de Junio. Dans ces configurations-là, la présence d'un robinet dans la maison n'empêche pas d'aller laver le linge ou la vaisselle dans un puits voisin, sauf dans les quelques cas où l'eau est assez abondante pour ne donner lieu à aucune dépense individuelle ou collective (à part la contribution des membres de la coopérative aux travaux d'installation et d'entretien). Si l'on cumule l'usage des puits et les connexions coopératives, on arrive à $89 \%$ de foyers étudiés qui ne sont pas connectés au réseau EPSAS. 6 \% ont accès à des bornes fontaines, le reliquat étant constitué par des familles qui vont chercher de l'eau par leurs propres moyens dans les sources à l'extérieur du quartier. En ce qui concerne les personnes reliées au réseau EPSAS, on peut constater que pour la plupart d'entre elles, la connexion date d'avant l'arrivée de l'entreprise Aguas del Illimani en 1997, et que la période 2001-2008 montre des taux particulièrement faibles de raccordement au réseau (I'année 2008 faisant exception, ce qui correspond à la mise en place de l'entreprise municipale).

Les chefs de famille sont à $80 \%$ des hommes, généralement mariés. La structure d'âge fait apparaître la relative absence de jeunes ménages $(13 \%$ de chefs de famille ont moins de 30 ans) au profit de couples d'âge moyen (31\% ont entre 30 et 39 ans, $25 \%$ entre 40 et 49 ans, soit $55 \%$ pour ces deux dernières catégories). Mais le fait que $32 \%$ des chefs de famille ait plus de 50 ans montre une plus grande diversité de peuplement que dans les zones périphériques de El Alto par exemple, où la grande majorité des coupes d'âge moyen renvoie à une migration intra-urbaine visant l'accès à la propriétél. Par ailleurs, $22 \%$ des

1 - Voir F. Poupeau : "Las desigualdades de acceso al agua en El Alto», Séminaire international sur « Modelos de gestión del agua en ciudades y comunidades de chefs de famille des zones étudiées vivent encore avec l'un des parents du couple, ce qui peut exprimer la persistance d'une transmission familiale située dans le quartier même. Parmi les ménages, $10 \%$ ont seulement un enfant, ce qui confirme la faible proportion de très jeunes couples; en revanche, $59 \%$ en ont plus de trois, indicateur caractéristique des milieux populaires boliviens. De même, seulement $10 \%$ des logements n'abritent qu'une ou deux personnes, $51 \%$ en abritent entre trois et cinq, et $39 \%$ en abritent plus de six, ce qui donne un nombre moyen de personnes par chambre compris entre 3 et 4 - un chiffre qui rapproche beaucoup plus les quartiers périphériques de La Paz du peuplement rural de Palca $(3,16)$ que de celui de la ville de La Paz $(2,36)$. L'organisation de l'habitat semble cependant moins précaire que dans d'autres quartiers périphériques comme ceux de El Alto : si $31 \%$ des logements ne comportent qu'une pièce (contre plus de $60 \%$ à El Alto), 32,5\% en ont plus de trois, et une grande majorité possède à la fois une cuisine séparée et des chambres exclusivement pour dormir.

Les transports occupent une place importante dans les budgets des familles : si $40 \%$ dépensent moins de 20 bolivianos (2 euros) par semaine (ce qui correspond au fait que $45 \%$ de l'échantillon travaille dans son quartier ou à moins d'une demi-heure de trajet), une grande partie des chefs de famille (37\%) dépensent entre 20 et 50 bolivianos par semaine, voire plus de 50 bolivianos pour le quart d'entre eux - $55 \%$ des chefs de famille ayant plus d'une heure de trajet pour aller à leur travail. On peut

los Andes», IFEA - PIEB, La Paz, 5-8 novembre 2007; «De la migración rural a la movilidad intra-urbana. Una perspectiva sociológica sobre las desigualdades socioespaciales de acceso al agua en El Alto, Bolivia», in F. Poupeau et C. Gonzales (dir.), Modelos de gestión del agua en los Andes, Lima, IFEA - PIEB, 2009. 
noter que seulement $10 \%$ d'entre eux ont une voiture, ce qui correspond principalement aux chauffeurs qui sont à leur compte propre, ou aux personnes résidant dans les condominiums de la zone sud de la ville. Du point de vue alimentaire, on constate là encore une certaine homogénéité : $80 \%$ de l'échantillon va une fois par semaine au marché, parfois quotidiennement à une épicerie de proximité pour des petites dépenses, mais en aucun cas aux supermarchés qui se sont développés sur La Paz depuis une quinzaine d'années, et dont les prix restent encore inabordables pour des ménages modestes. Si l'on s'en tient aux dépenses alimentaires déclarées, les quartiers périphériques de La Paz ont un niveau de vie supérieur à celui constaté à El Alto: seuls $20 \%$ des ménages consomment moins de 100 bolivianos par semaine pour la nourriture (et les tableaux croisés révèlent qu'il s'agit de familles restreintes), tandis que plus de la moitié de l'échantillon dépense plus de 150 bolivianos par semaine (les différences entre ménages dépendant du nombre d'enfants dans la famille). II est à signaler que ces chiffres doivent être maniés avec prudence, I'autoévaluation des dépenses n'étant pas toujours fiable: elle ne fait que révéler de grandes tendances, notamment d'un point de vue comparatif avec El Alto, où la moyenne des dépenses par semaine oscille plutôt entre 50 et 100 bolivianos $^{2}$. Certaines similarités existent néanmoins entre les quartiers périphériques des deux villes de I'agglomération: comme sur El Alto, une grande partie des familles (80\%) a l'intention d'agrandir sa maison dans le futur, notamment pour y ouvrir une épicerie ou garder du bétail.

Le fait que $85 \%$ des personnes interrogées à La $\mathrm{Paz}$ s'identifie à un «peuple originaire», princi-

2 - F. Poupeau, "Las desigualdades de acceso al agua en El Alto», conférence citée. palement aymara, révèle là encore une implantation plus rurale que dans le centre de La Paz (64 \%) et même que El Alto (81 \%), souvent qualifiée de "cité aymara ». Cette hypothèse est confirmée par la profession des parents : $52 \%$ des parents des chefs de famille interrogés sont agriculteurs, contre $16 \%$ maçons, $4 \%$ chauffeurs, $6 \%$ commerçants; les parents appartenant à des "professions supérieures» représentent $5 \%$ de l'échantillon, ce qui concorde avec la présence d'enclaves plus privilégiées dans certains quartiers, comme Chicani ou Chijipata. Du point de vue socioprofessionnel, les chefs de famille se situent plutôt au plus bas de la hiérarchie sociale, excepté dans les quartiers de Chijipata et des condominiums. Les travailleurs non qualifiés, qu'il s'agisse des maçons ou des ouvriers industriels, représentent $38 \%$ de l'échantillon; $28 \%$ des chefs de famille sont dans le commerce et les services, $9 \%$ sont agriculteurs, $7 \%$ employés de bureau, et $10 \%$ professions supérieures (direction d'entreprise, professions scientifiques et techniques), tous regroupés dans les condominiums ou le quartier de Chijipata. Parmi les chefs de famille féminins (20\% de l'échantillon), la moitié d'entre elles restent travailler à domicile, la plupart du temps à exploiter un petit lopin de terre assurant à peine I'autosubsistance: comme sur El Alto, il s'agit des foyers les plus en difficulté. Par rapport aux parents des chefs de famille de l'échantillon, on note malgré tout une légère mobilité ascendante, avec une réduction de la proportion d'agriculteurs et une augmentation de professions intermédiaires ou supérieures, liées au développement des services dans l'agglomération de La Paz.

Du point de vue du statut, les deux tiers des chefs de famille de l'échantillon se déclarent salariés, de façon plus ou moins permanente; $26 \%$ d'entre eux sont à compte propre, mais seulement $3 \%$ se déclarent véritablement entrepreneurs, ce qui recouvre grosso modo 
les données de l'agglomération de La Paz ${ }^{3}$ La population de ces quartiers périphériques est donc de milieu populaire, mais elle ne condense pas non plus les fractions les plus marginales de la population, comme on pourrait I'attendre du type de lieux habités. En revanche, les résidents restent, comme la plupart des travailleurs de La Paz et de El Alto, dans des situations précaires, comme le montrent le faible taux de personnes possédant une assurance personnelle (19\%), mais aussi l'examen des secteurs d'activité : $30 \%$ de l'échantillon travaille dans la construction (secteur d'emplois journaliers et faiblement contractualisés), contre $13 \%$ dans le commerce, $15 \%$ dans les transports, $10 \%$

3 - À Palca en revanche, où la majorité de la population active exerce le métier d'agriculteur, le taux de travailleurs à compte propre est de $70 \%$. dans le travail à domicile (essentiellement des femmes), $9 \%$ dans I'agriculture, $7 \%$ dans la manufacture, et seulement $4 \%$ dans les diverses administrations de services urbains et l'éducation. Le personnel de santé représente $10 \%$ de l'échantillon, mais s'agissant du secteur public, ce sont sans doute des infirmiers ou employé(e)s sous-payés, au même titre que les enseignants du primaire. Cette faible qualification des emplois se retrouve avec le niveau d'instruction, plus proche cependant de celui de la population de La Paz que celui des habitants de Palca : si près de $10 \%$ des chefs de famille sont analphabètes (contre $7 \%$ à La Paz et $22 \%$ à Palca), $42 \%$ ont arrêté leurs études au niveau primaire (respectivement $24 \%$ et $62 \%$ ), $35 \%$ dans le secondaire (respectivement $34 \%$ et $8 \%$ ), mais $9 \%$ seulement ont été jusqu'à l'université (respectivement $30 \%$ et moins de $2 \%$ ).

parmi les fractions populaires et économiquement modestes de la population. On peut alors s'attendre à ce que le décalage de cette situation que l'on pourrait qualifier de "moyenne inférieure» avec le sous-équipement des quartiers habités soit générateur de frustrations voire de conflits. La caractérisation des conditions de vie matérielles et des propriétés sociales des chefs de famille montre que les quartiers périphériques de La Paz sont en effet composés de familles trop modestes pour accéder à la propriété dans des zones plus centrales, et qui acceptent pour un temps indéterminé de ne pas bénéficier de tous les services urbains auxquels elles aspirent. Ainsi les demandes de connexion au réseau de distribution d'eau font apparaître que, comme à El Alto ${ }^{12}$, aucune réticence majeure n'existe concernant le paiement mensuel de la facture d'eau : les exemples recueillis de gens revendiquant la gratuité de la ressource correspondent à la commune de Chicani, où un système communautaire fournit de l'eau de source en abondance à quelques blocs de maisons. Dans ces cas-là (qui représentent $10 \%$ de l'échantillon), les habitants concernés affirment ne pas vouloir se connecter au réseau, alors que la majorité des autres enquêtés expriment un fort désir de connexion. Il y a sur ce point un biais qui tient aux conditions d'enquête par questionnaire : les personnes n'étant pas intéressées

12 - F. Poupeau, "Las desigualdades de acceso al agua en El Alto», séminaire international sur "Modelos de gestión del agua en ciudades y comunidades de los Andes», Institut français d'études andines (IFEA) - Programa de Investigación Estratégica en Bolivia (PIEB), La Paz, 5-8 novembre 2007. 
par une connexion, comme les agriculteurs de quartiers frontaliers entre La Paz et les communes voisines, désireux de ne pas être rattachés à La Paz pour ne pas payer les impôts locaux sur les terres plus élevés qui y ont cours (par exemple Apaña), n'ont pas voulu répondre au questionnaire, ou bien n'ont pas pu le faire en raison de leur absence quasi permanente du domicile, voire pour quelques cas à cause de l'obstacle linguistique que représentait pour eux la communication en espagnol.

La population des quartiers non équipés de La Paz s'avère en outre beaucoup moins mobilisée sur le thème de l'eau que celle de El Alto, épicentre des mobilisations pour le retour à la nationalisation des ressources naturelles entre 2003 et $2005^{13}$. Certes, près de la moitié de l'échantillon estime que l'absence d'eau a constitué un problème lors de l'installation dans la résidence actuelle, mais $53 \%$ des personnes interrogées seulement connaissent le nom et l'existence de l'entreprise EPSAS, et moins de $40 \%$ ont une opinion déclarée sur les effets bénéfiques ou non du départ de l'entreprise Aguas del Illimani. L'eau ne semble pas perçue comme un thème politique alors que la grande majorité des habitants (90\%) se déclare prête à faire des dépenses pour accéder au réseau. Lorsqu'ils prennent connaissance des prix de connexion (seulement $17 \%$ des personnes interrogées ont pu dire quel était le tarif), les pourcentages retombent quelque peu : $76 \%$ et $67 \%$ sont prêts à débourser respectivement 150 dollars pour l'eau potable et 190 dollars pour l'assainissement. Alors que sur les prix de consommation, les opinions sont partagées (31 \% les trouvent trop chers, $32 \%$ les estiment raisonnables et $37 \%$ sont sans opinion), les prix de connexion sont estimés trop chers par $61 \%$ de l'échantillon : là encore, il semble que la population de ces quartiers connaisse moins de difficultés économiques que dans les quartiers en expansion de El Alto (où ce taux dépasse les $90 \%$ ). Par ailleurs, le problème du raccordement au réseau est perçu comme un problème relevant moins de l'individuel que du collectif : la connexion à domicile n'est pensée, dans tous les cas, que dans le cadre d'une connexion de l'ensemble du voisinage. On voit bien ici ce qui sépare, aussi bien au niveau des conditions matérielles que des façons de penser, les habitants de ces quartiers en extension des "usagers» que la science économique et la sociologie des organisations de services urbains mettent au centre de leurs préceptes normatifs - surtout lorsqu'il s'agit d'équiper les villes dites « du Sud».

La perception des problèmes d'accès à l'eau et les dispositions à se connecter au réseau font cependant apparaître des différences substantielles selon les quartiers. Dans plusieurs des zones étudiées, le prix de connexion individuelle ne correspond pas à la somme que devraient débourser les habitants pour se raccorder : situés en hauteur, engoncés entre des pics rocheux qui constituent autant d'obstacles naturels, ils devraient faire installer des systèmes plus coûteux, destinés à compenser le manque de pression. Moins en difficulté financière que les habitants de El Alto, les résidents trouvent dans les conditions spatiales de leur installation un facteur d'inégalité d'accès au service : les plus pauvres résident généralement dans les secteurs les plus difficiles d'accès, les plus éloignés du centre et les plus proches des montagnes

13 - F. Poupeau, «El Alto : una ficción política», conférence citée. 
environnantes. Par ailleurs, l'échantillon étudié fait apparaître une image relativement précise de l'hétérogénéité des conditions d'accès au service d'eau dans les quartiers périphériques de La Paz (voir ci-dessous les tableaux sur les différenciations par quartiers). Chaque variable prise séparément ne permet cependant pas de rendre compte des inégalités entre quartiers : il n'y a pas d'échelle unique permettant de les ordonner en faisant correspondre le niveau d'équipement en services urbains de la zone considérée, le type d'habitations, avec les caractéristiques démographiques, sociales et économiques des familles. Des quartiers composés de familles ayant la capacité de payer la connexion au réseau rencontrent plus de difficultés à s'équiper à cause d'obstacles physiques, d'une distance trop grande au réseau ou d'installation trop récente. Il faut donc croiser variables sociales et spatiales pour rendre compte des disparités rencontrées: dans cette perspective, on fait ici l'hypothèse que les différences entre les quartiers en termes d'accès au service de distribution des eaux constituent un indicateur sociospatial plus précis que les seuls indicateurs de localisation de la résidence ou la seule profession des chefs de famille. Afin de prendre en compte les différences de situations rencontrées, une analyse multidimensionnelle des questionnaires a donc été réalisée sur la base territoriale des quartiers.

\section{DÉTAILS DE LA DIFFÉRENCIATION SOCIOSPATIALE ENTRE QUARTIERS}

1 - FORMES D'ACCÈS À L'EAU POTABLE

\begin{tabular}{|c|c|c|c|c|}
\hline & 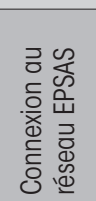 & 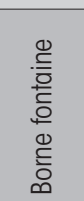 & 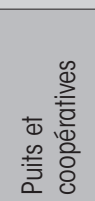 & 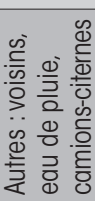 \\
\hline Chicani & $5,7 \%$ & $2,9 \%$ & $77,1 \%$ & $14,7 \%$ \\
\hline Apaña & $3,6 \%$ & $3,6 \%$ & $25,0 \%$ & $67,9 \%$ \\
\hline 24 de Junio & $4,3 \%$ & $8,7 \%$ & $43,5 \%$ & $43,5 \%$ \\
\hline Pokechaka & - & - & $88,5 \%$ & $11,5 \%$ \\
\hline Chijipata \& co & $15,0 \%$ & - & $50,0 \%$ & $35,0 \%$ \\
\hline Mirador & $8,3 \%$ & $25 \%$ & $8,3 \%$ & $58,3 \%$ \\
\hline Alto Ovejuyo & & - & $91,7 \%$ & $8,3 \%$ \\
\hline
\end{tabular}

2 - PÉriode d'installation dans le Quartier

\begin{tabular}{|c|c|c|c|c|}
\hline & 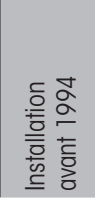 & 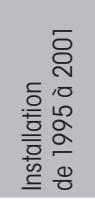 & 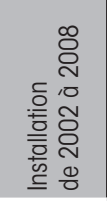 & 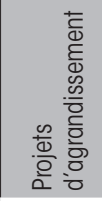 \\
\hline Chicani & $71,9 \%$ & $12,5 \%$ & $15,6 \%$ & $66,7 \%$ \\
\hline Apaña & $53,6 \%$ & $10,7 \%$ & $35,7 \%$ & $62,5 \%$ \\
\hline 24 de Junio & $60,9 \%$ & $30,4 \%$ & $8,7 \%$ & $86,4 \%$ \\
\hline Pokechaka & $46,2 \%$ & $34,6 \%$ & $19,2 \%$ & $94,4 \%$ \\
\hline Chijipata \& co & $21,1 \%$ & $15,8 \%$ & $63,2 \%$ & $82,4 \%$ \\
\hline Mirador & & - & $100,0 \%$ & $100,0 \%$ \\
\hline Alto Ovejuyo & $16,7 \%$ & $41,7 \%$ & $41,7 \%$ & $83,3 \%$ \\
\hline
\end{tabular}

3 - CARActéRISTIQUeS deS CheFs de FAMILLE

\begin{tabular}{|c|c|c|c|c|c|c|c|c|c|}
\hline & 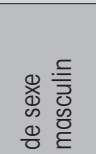 & $\begin{array}{l}\text { ¿ै } \\
\text { Oे } \\
\text { gे } \\
\text { v }\end{array}$ & $\begin{array}{l}\text { こ } \\
0 \\
0 \\
1 \\
\wedge\end{array}$ & 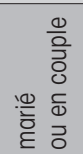 & 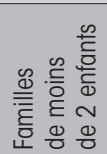 & 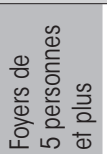 & 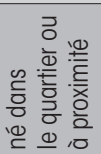 & 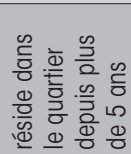 & 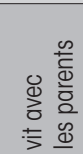 \\
\hline Chicani & $88,6 \%$ & $44,1 \%$ & $20,6 \%$ & $75,8 \%$ & $38,2 \%$ & $48,7 \%$ & $61,8 \%$ & $87,9 \%$ & $27,3 \%$ \\
\hline Apaña & $89,3 \%$ & $33,3 \%$ & $14,8 \%$ & $89,3 \%$ & $37,0 \%$ & $39,1 \%$ & $53,6 \%$ & $85,2 \%$ & $14,8 \%$ \\
\hline 24 de Junio & $73,9 \%$ & $47,8 \%$ & $8,6 \%$ & $88,9 \%$ & $34,7 \%$ & $61,8 \%$ & $4,3 \%$ & $90,5 \%$ & $26,1 \%$ \\
\hline Pokechaka & $75,0 \%$ & $44,0 \%$ & $4.0 \%$ & $84,6 \%$ & $36,0 \%$ & $51,7 \%$ & $26,9 \%$ & $82,6 \%$ & $16,7 \%$ \\
\hline Chijipata \& co & $68,4 \%$ & $29,4 \%$ & $23,6 \%$ & $68,4 \%$ & $42,1 \%$ & $65,0 \%$ & $51,0 \%$ & $73,7 \%$ & $21,1 \%$ \\
\hline Mirador & $66,7 \%$ & $41,7 \%$ & $8,7 \%$ & $80,0 \%$ & $63,6 \%$ & $36,2 \%$ & $50,0 \%$ & $90,9 \%$ & - \\
\hline Alto Ovejuyo & $83,3 \%$ & $83,4 \%$ & $4,3 \%$ & $100 \%$ & $49,9 \%$ & $74,8 \%$ & $41,7 \%$ & $91,7 \%$ & $41,7 \%$ \\
\hline
\end{tabular}


4 - Statut des ChefS de famille

\begin{tabular}{|l|c|c|c|c|c|c|}
\cline { 2 - 7 } & $\begin{array}{c}\text { Ouvriers } \\
\text { et employés } \\
\text { salariés }\end{array}$ & $\begin{array}{c}\text { Travailleurs } \\
\text { à compte } \\
\text { propre }\end{array}$ & $\begin{array}{c}\text { Patrons } \\
\text { et employeurs }\end{array}$ & $\begin{array}{c}\text { Travailleurs } \\
\text { de coopératives }\end{array}$ & $\begin{array}{c}\text { Travailleurs } \\
\text { à domcile sans } \\
\text { rémunération }\end{array}$ & $\begin{array}{c}\text { Possède } \\
\text { une assurance } \\
\text { personnelle }\end{array}$ \\
\hline Chicani & $53,1 \%$ & $31,3 \%$ & $9,4 \%$ & $6,3 \%$ & - & $12,1 \%$ \\
\hline Apaña & $69,2 \%$ & $30,8 \%$ & - & - & - & $17,9 \%$ \\
\hline 24 de Junio & $70,0 \%$ & $25,0 \%$ & - & - & $5,0 \%$ & $30,4 \%$ \\
\hline Pokechaka & $87,0 \%$ & $13,0 \%$ & - & - & - & $7,7 \%$ \\
\hline Chijipata \& co & $57,9 \%$ & $31,6 \%$ & $5,3 \%$ & - & $5,3 \%$ & $44,4 \%$ \\
\hline Mirador & $75,0 \%$ & $25,0 \%$ & - & - & - & - \\
\hline Alto Ovejuyo & $72,7 \%$ & $18,2 \%$ & - & - & $9,1 \%$ & $25,0 \%$ \\
\hline
\end{tabular}

\section{5 - Profession des Chefs de famille}

\begin{tabular}{|l|c|c|c|c|c|c|}
\cline { 2 - 7 } & $\begin{array}{c}\text { Professions } \\
\text { supérieures } \\
\text { et techniques } \\
\text { qualifiées }\end{array}$ & $\begin{array}{c}\text { Employés } \\
\text { de bureau }\end{array}$ & Commerçants & Agriculteurs & $\begin{array}{c}\text { Ouvriers non qualifiés } \\
\text { (construction, } \\
\text { industrie, transports) }\end{array}$ & $\begin{array}{c}\text { Autres } \\
\text { (retraités, } \\
\text { femmes } \\
\text { au foyer) }\end{array}$ \\
\hline Chicani & $8,8 \%$ & $2,9 \%$ & $17,6 \%$ & $29,4 \%$ & $35,2 \%$ & $5,8 \%$ \\
\hline Apaña & $7,2 \%$ & $3,6 \%$ & $35,7 \%$ & $10,7 \%$ & $35,8 \%$ & $7,2 \%$ \\
\hline 24 de Junio & $16,9 \%$ & $17,8 \%$ & $34,8 \%$ & - & $26,1 \%$ & $4,3 \%$ \\
\hline Pokechaka & - & $8 \%$ & $32,0 \%$ & - & $44,0 \%$ & $16,0 \%$ \\
\hline Chijipata \& co & $21,1 \%$ & $10,5 \%$ & $32,2 \%$ & - & $31,0 \%$ & $5,3 \%$ \\
\hline Mirador & - & $9,1 \%$ & $27,3 \%$ & - & $63,6 \%$ & - \\
\hline Alto Ovejuyo & - & - & $16,9 \%$ & - & $66,5 \%$ & $16,5 \%$ \\
\hline
\end{tabular}

6 - Profession du père du Chef de famille

\begin{tabular}{|l|c|c|c|c|c|c|}
\cline { 2 - 7 } & $\begin{array}{c}\text { Professions } \\
\text { supérieures } \\
\text { et techniques } \\
\text { qualifiées }\end{array}$ & $\begin{array}{c}\text { Employés } \\
\text { de bureau }\end{array}$ & Commerçants & Agriculteurs & $\begin{array}{c}\text { Ouvriers non qualifiés } \\
\text { (construction, } \\
\text { industrie, transports) }\end{array}$ & $\begin{array}{c}\text { Autres } \\
\text { (retraités...) }\end{array}$ \\
\hline Chicani & $5,7 \%$ & $5,7 \%$ & - & $71,4 \%$ & $8,8 \%$ & $8,6 \%$ \\
\hline Apaña & $3,8 \%$ & $3,8 \%$ & $15,4 \%$ & $46,2 \%$ & $5,0 \%$ & $7,7 \%$ \\
\hline 24 de Junio & & $23,5 \%$ & $5,9 \%$ & $52,9 \%$ & $5,9 \%$ & $11,8 \%$ \\
\hline Pokechaka & - & $7,7 \%$ & $53,8 \%$ & $23,1 \%$ & $3,8 \%$ \\
\hline Chijipata \& co & $83,7 \%$ & $10,4 \%$ & $13,5 \%$ & - & - & $3,4 \%$ \\
\hline Mirador & - & $11,0 \%$ & $15,0 \%$ & $41,5 \%$ & $22,0 \%$ & $11,5 \%$ \\
\hline Alto Ovejuyo & - & - & - & $40,0 \%$ & $20,0 \%$ & $20,0 \%$ \\
\hline
\end{tabular}

7 - Niveau d'instruction du Chef de famille

\begin{tabular}{|l|c|c|c|c|}
\cline { 2 - 5 } \multicolumn{1}{c|}{} & Aucun & Primaire & Secondaire & Supérieur \\
\hline Chicani & $8,8 \%$ & $61,8 \%$ & $14,7 \%$ & $14,7 \%$ \\
\hline Apaña & $10,7 \%$ & $53,6 \%$ & $28,6 \%$ & $7,1 \%$ \\
\hline 24 de Junio & $9,1 \%$ & $36,4 \%$ & $50,0 \%$ & $4,5 \%$ \\
\hline Pokechaka & $11,5 \%$ & $38,5 \%$ & $42,3 \%$ & $7,7 \%$ \\
\hline Chijipata \& co & $10,0 \%$ & $30,0 \%$ & $40,0 \%$ & $20,0 \%$ \\
\hline Mirador & - & $63,6 \%$ & $36,4 \%$ & - \\
\hline Alto Ovejuyo & - & $41,7 \%$ & $58,3 \%$ & - \\
\hline
\end{tabular}


8 - Motifs d'INSTALLATION DANS LE QUARTIER

(DONNÉES PORTANT SUR LA FRACTION MIGRANTE DE LA POPULATION ÉTUDIÉE)

\begin{tabular}{|c|c|c|c|c|c|c|c|}
\hline & 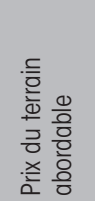 & 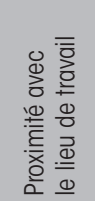 & 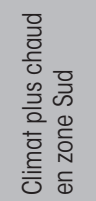 & 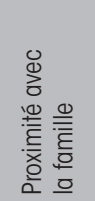 & 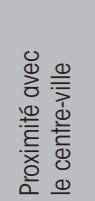 & 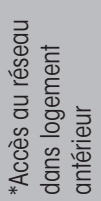 & 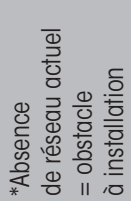 \\
\hline Chicani & - & $66,7 \%$ & $33,3 \%$ & - & - & $61,5 \%$ & $66,7 \%$ \\
\hline Apaña & $13,0 \%$ & $8,7 \%$ & $8,7 \%$ & $47,8 \%$ & $17,4 \%$ & $50,0 \%$ & $77,8 \%$ \\
\hline 24 de Junio & $10,0 \%$ & $45,0 \%$ & $20,0 \%$ & $20,0 \%$ & $5,0 \%$ & $26,7 \%$ & $52,2 \%$ \\
\hline Pokechaka & $5,3 \%$ & $41,1 \%$ & $22,1 \%$ & $10,5 \%$ & $21,1 \%$ & $55,6 \%$ & $84,0 \%$ \\
\hline Chijipata \& co & $45,2 \%$ & - & $28,8 \%$ & - & $25,0 \%$ & $66,7 \%$ & $69,2 \%$ \\
\hline Mirador & 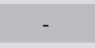 & $60,0 \%$ & $30,0 \%$ & - & $10,0 \%$ & $44,4 \%$ & $66,7 \%$ \\
\hline Alto Ovejuyo & - & $50,0 \%$ & $20,0 \%$ & $30,0 \%$ & - & $66,7 \%$ & $58,3 \%$ \\
\hline
\end{tabular}

9 - TEMPS DE TRANSPORT ENTRE LE DOMICILE ET LE LIEU DE TRAVAIL

\begin{tabular}{|c|c|c|c|c|c|c|}
\hline & 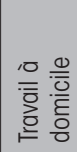 & 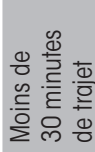 & 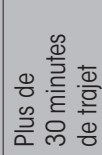 & 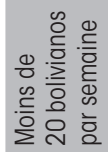 & 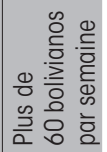 & 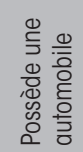 \\
\hline Chicani & $4,3 \%$ & $50,9 \%$ & $34,7 \%$ & $58,1 \%$ & $27,1 \%$ & $27,3 \%$ \\
\hline Apaña & - & $47,1 \%$ & $53,0 \%$ & $41,2 \%$ & $5,9 \%$ & $3,6 \%$ \\
\hline 24 de Junio & - & $35,3 \%$ & $64,7 \%$ & $28,1 \%$ & $23,9 \%$ & $8,7 \%$ \\
\hline Pokechaka & $9,1 \%$ & $9,1 \%$ & $81,8 \%$ & $22,7 \%$ & $22,6 \%$ & - \\
\hline Chijipata\&co & - & $53,3 \%$ & $23,6 \%$ & $23,1 \%$ & $15,4 \%$ & $26,3 \%$ \\
\hline Mirador & - & $30,0 \%$ & $70,0 \%$ & $45,5 \%$ & $36,4 \%$ & - \\
\hline Alto Ovejuyo & - & $50,0 \%$ & 50,0 & $41,6 \%$ & $25,0 \%$ & $16,7 \%$ \\
\hline
\end{tabular}

10 - Évaluation des dÉPENSES HEBDOMADAIRES AU MARCHÉ

\begin{tabular}{|c|c|c|}
\hline 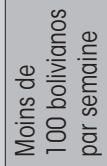 & 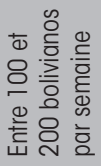 & 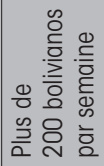 \\
\hline $15,6 \%$ & $50,1 \%$ & $34,3 \%$ \\
\hline $20,0 \%$ & $48,0 \%$ & $32,0 \%$ \\
\hline $25,0 \%$ & $35,2 \%$ & $38,8 \%$ \\
\hline $29,2 \%$ & $37,5 \%$ & $33,2 \%$ \\
\hline $12,6 \%$ & $56,4 \%$ & $31,3 \%$ \\
\hline $16,9 \%$ & $66,6 \%$ & $16,5^{\circ}$ \\
\hline $18,2 \%$ & $54,6 \%$ & $27,3^{\circ}$ \\
\hline
\end{tabular}

11 - CONDITIONS D'HYGIENE

\begin{tabular}{|c|c|c|c|c|c|c|c|}
\hline & 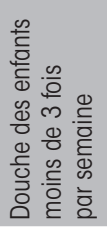 & 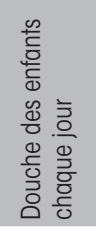 & 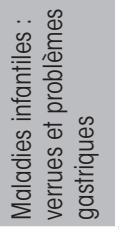 & 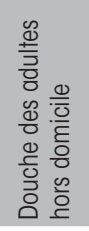 & 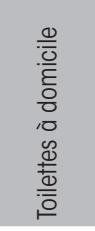 & 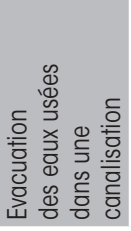 & 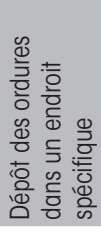 \\
\hline Chicani & $75,0 \%$ & $25,0 \%$ & $48,4 \%$ & $31,4 \%$ & $44,1 \%$ & $3,2 \%$ & $87,1 \%$ \\
\hline Apaña & $88,8 \%$ & $29,2 \%$ & $34,6 \%$ & $50,0 \%$ & $28,6 \%$ & - & $80,0 \%$ \\
\hline 24 de Junio & $80,0 \%$ & $20,0 \%$ & $21,3 \%$ & $69,6 \%$ & $43,5 \%$ & $5,0 \%$ & $93,8 \%$ \\
\hline Pokechaka & $81,0 \%$ & $19,0 \%$ & $41,8 \%$ & $52,0 \%$ & - & - & $71,4 \%$ \\
\hline Chijipata\&co & $72,7 \%$ & $27,3 \%$ & $32,5 \%$ & $66,7 \%$ & $68,4 \%$ & $20,0 \%$ & $94,7 \%$ \\
\hline Mirador & $90,0 \%$ & $10,0 \%$ & $35,9 \%$ & $90,0 \%$ & - & - & $27,3 \%$ \\
\hline Alto Ovejuyo & $81,8 \%$ & $18,2 \%$ & $50,0 \%$ & $83,3 \%$ & - & - & $72,7 \%$ \\
\hline
\end{tabular}


12 - Prises de position envers le service de distribution des eauX

a) la connexion au réseau

\begin{tabular}{|l|c|c|c|c|c|c|}
\cline { 2 - 7 } & $\begin{array}{c}\text { Connaissance } \\
\text { des prix } \\
\text { de connexion } \\
\text { au réseau }\end{array}$ & $\begin{array}{c}\text { Tarifs } \\
\text { de connexion } \\
\text { estimés } \\
\text { raisonnables }\end{array}$ & $\begin{array}{c}\text { Tarifs } \\
\text { de connexion } \\
\text { estimés } \\
\text { trop chers }\end{array}$ & $\begin{array}{c}\text { Sans opinion } \\
\text { sur les prix } \\
\text { de connexion }\end{array}$ & $\begin{array}{c}\text { Prêts à } \\
\text { se connecter } \\
\text { à l'eau potable } \\
(150 \text { \$) }\end{array}$ & $\begin{array}{c}\text { Prêts à } \\
\text { l'assainissement } \\
(185 \text { \$) }\end{array}$ \\
\hline Chicani & $3,0 \%$ & $15,2 \%$ & $72,7 \%$ & $12,1 \%$ & $51,6 \%$ & $45,2 \%$ \\
\hline Apaña & $8,0 \%$ & $11,5 \%$ & $80,8 \%$ & $7,7 \%$ & $80,8 \%$ & $57,7 \%$ \\
\hline 24 de Junio & $17,4 \%$ & $39,1 \%$ & $56,5 \%$ & $4,3 \%$ & $90,9 \%$ & $78,3 \%$ \\
\hline Pokechaka & $12,0 \%$ & $4,0 \%$ & $80,0 \%$ & $16,0 \%$ & $76,0 \%$ & $60,0 \%$ \\
\hline Chijipata \& co & $52,9 \%$ & $64,7 \%$ & $17,1 \%$ & $18,1 \%$ & $93,8 \%$ & $87,5 \%$ \\
\hline Mirador & $16,7 \%$ & $12,5 \%$ & $25,0 \%$ & $62,5 \%$ & $66,7 \%$ & $41,7 \%$ \\
\hline Alto Ovejuyo & $33,3 \%$ & $33,3 \%$ & $41,7 \%$ & $25,0 \%$ & $83,3 \%$ & $41,7 \%$ \\
\hline
\end{tabular}

b) la consommation

\begin{tabular}{|l|c|c|c|c|}
\cline { 2 - 4 } & $\begin{array}{c}\text { Estime normal de payer } \\
\text { la consommation d'eau }\end{array}$ & $\begin{array}{c}\text { Tarifs de } \\
\text { consommation } \\
\text { estimés raisonnables }\end{array}$ & $\begin{array}{c}\text { Tarifs de } \\
\text { consommation } \\
\text { estimés trop chers }\end{array}$ & $\begin{array}{c}\text { Sans opinion } \\
\text { sur les tarifs } \\
\text { de consommation }\end{array}$ \\
\hline Chicani & $78,1 \%$ & $36,4 \%$ & $48,5 \%$ & $15,2 \%$ \\
\hline Apaña & $91,3 \%$ & $43,5 \%$ & $8,7 \%$ & $47,8 \%$ \\
\hline 24 de Junio & $95,5 \%$ & $30,4 \%$ & $34,8 \%$ & $34,8 \%$ \\
\hline Pokechaka & $93,3 \%$ & $22,7 \%$ & $36,4 \%$ & $40,9 \%$ \\
\hline Chijipata \& co & $100,0 \%$ & $52,9 \%$ & $17,6 \%$ & $29,4 \%$ \\
\hline Mirador & $83,3 \%$ & $14,3 \%$ & $28,6 \%$ & $57,1 \%$ \\
\hline Alto Ovejuyo & $91,7 \%$ & - & $33,3 \%$ & $66,7 \%$ \\
\hline
\end{tabular}

* Ci-contre (tabl. 13), I'absence de réponses à Mirador Turístico sur les trois dernières entrées tient au fait que le quartier est démuni d'un quelconque approvisionnement en eau trouvant sa source sur place.

\section{Intégration socioéconomique et accessibilité spatiale}

En partant des conditions sociales d'accès à l'eau dans les quartiers les moins équipés, l'enquête présentée ici se démarque des approches politistes, qui se concentrent sur l'organisation institutionnelle des services urbains. Elle se différencie aussi des approches géographiques, qui s'appuient avant tout sur le repérage des spécialisations spatiales. La mesure de l'intensité de la spécialisation d'un espace donné suppose en effet une «interdépendance des unités spatiales entre elles $^{14}$ ", dans un espace de référence donné, pour un domaine particulier (type d'activité, forme du travail, appartenance sociale de la population résidente, etc.) ; elle utilise pour cela des tableaux d'information géographique qui prennent la forme de tableaux de contingence croisant les unités spatiales et le domaine de

14 - D. Pumain et T. Saint-Julien, Les interactions spatiales: flux et changements dans l'espace géographique, Paris, Armand Colin, 2001, p. 60-62. 
c) le passage du privé au public

\begin{tabular}{|c|c|c|c|c|c|}
\hline & 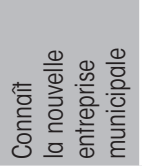 & 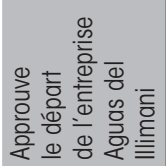 & 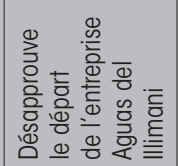 & 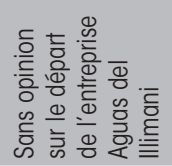 & 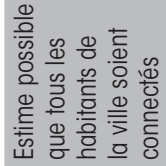 \\
\hline Chicani & $58,6 \%$ & $34,8 \%$ & - & $65,2 \%$ & $58,1 \%$ \\
\hline Apaña & $53,8 \%$ & $45,9,0 \%$ & - & $54,1 \%$ & $69,2 \%$ \\
\hline 24 de Junio & $73,9 \%$ & $81,0 \%$ & - & $19,0 \%$ & $65,0 \%$ \\
\hline Pokechaka & $64,0 \%$ & $65,0 \%$ & $10,0 \%$ & $25,0 \%$ & $70,8 \%$ \\
\hline Chijipata\&co & $75,0 \%$ & $40,0 \%$ & $20,0 \%$ & $40,0 \%$ & $71,4 \%$ \\
\hline Mirador & $36,4 \%$ & $42,1 \%$ & $11,1 \%$ & $46,8 \%$ & $20,0 \%$ \\
\hline Alto Ovejuyo & $35,0 \%$ & $55,6 \%$ & $10,8 \%$ & $33,6 \%$ & $60,0 \%$ \\
\hline
\end{tabular}

13 - Perceptions des RISQues ENVIRONNementauX

\begin{tabular}{|c|c|c|c|c|c|c|c|c|}
\hline & 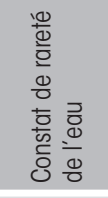 & 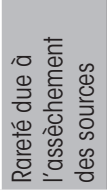 & 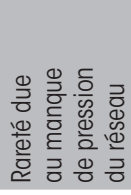 & 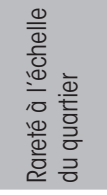 & 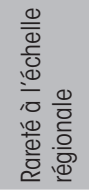 & 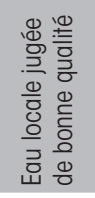 & 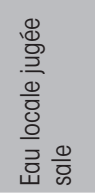 & 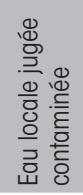 \\
\hline Chicani & $80,6 \%$ & $50,0 \%$ & - & $50,0 \%$ & $59,3 \%$ & $53,9 \%$ & $26,9 \%$ & $19,2 \%$ \\
\hline Apaña & $96,2 \%$ & $69,2 \%$ & $7,7 \%$ & $23,1 \%$ & $91,7 \%$ & $47,8 \%$ & $26,1 \%$ & $26,1 \%$ \\
\hline 24 de Junio & $87,0 \%$ & $80,0 \%$ & $10,0 \%$ & $10,0 \%$ & $76,2 \%$ & $33,3 \%$ & $19,0 \%$ & $47,6 \%$ \\
\hline Pokechaka & $100,0 \%$ & $41,2 \%$ & $5,9 \%$ & $52,9 \%$ & $57,1 \%$ & $24,0 \%$ & $8,0 \%$ & $68,0 \%$ \\
\hline Chijipata \& co & $92,9 \%$ & - & - & $100,0 \%$ & $46,2 \%$ & $20,0 \%$ & $40,0 \%$ & $40,0 \%$ \\
\hline Mirador & $71,4 \%$ & 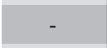 & - & $50,0 \%$ & $66,7 \%$ & -* & -* & -* \\
\hline Alto Ovejuyo & $100,0 \%$ & $100 \%$ & - & $23,1 \%$ & $90,0 \%$ & & - & $100 \%$ \\
\hline
\end{tabular}

spécialisation. L'enquête sur les inégalités sociospatiales d'accès à l'eau menée à La Paz s'inscrit dans une perspective quelque peu différente: elle consiste à étudier non pas un domaine d'activité spécifique mais les relations entre les différentes dimensions de la vie sociale associées aux unités spatiales étudiées. Cette démarche utilise donc des analyses factorielles qui ont pour but de mettre en relation les différents types de variables sociales, économiques, démographiques, spatiales, organisationnelles ou institutionnelles : les axes principaux permettent alors d'identifier des indicateurs pertinents pour rendre compte des inégalités observées ${ }^{15}$. L'analyse des correspondances menée à partir du codage des questionnaires utilise, en variables principales, les caractéristiques sociales des chefs de famille, les caractéristiques spatiales des quartiers, des logements et des conditions d'accès à l'eau, et en variables supplémentaires la perception du service par les

15 - F. Lebaron, L'enquête quantitative en sciences sociales. Recueil et analyse des données, Paris, Dunod, 2006. 
résidents et leurs demandes en termes de services urbains. L'espace des quartiers considérés se révèle alors structuré par une double opposition. Le premier axe (58\% de la variance) distingue les quartiers en fonction du degré d'intégration socioéconomique des habitants, mesuré à l'importance de la profession du chef de famille, à sa stabilité en termes de revenus et à sa participation au secteur formel du marché du travail. Le second axe (42\% de la variance) exprime le degré d'accessibilité matérielle du quartier au réseau des services urbains, en fonction de la distance avec le centre-ville, de la présence d'obstacles physiques et d'une voirie en bon état, reconnue par les autorités publiques (et sur laquelle transitent des moyens de locomotion collectifs plus ou moins nombreux et réguliers).

Sur le plan formé par les axes 1 et 2 (voir schémas 1 et 2), on peut distinguer (en haut à droite) les quartiers en voie d'équipement (Chijipata, condominiums de Cota Cota) qui réunissent à la fois le plus de facilités matérielles d'accès au service de distribution des eaux (faible éloignement avec le centre-ville, peu d'obstacles géographiques, transports en commun fréquents) et le plus de moyens chez les habitants, non seulement en termes purement économiques, mais aussi en termes de capacités à réaliser les démarches administratives auprès de l'entreprise d'eau ou de la mairie. Ces quartiers se distinguent des autres aussi bien par les caractéristiques de la population que par celles du logement. Ils sont les seuls de l'échantillon à compter, parmi leurs résidents, une fraction non négligeable de chefs de famille appartenant aux professions supérieures, et ils sont surtout composés de professions intermédiaires (employés) et de petits entrepreneurs (artisanat, commerce, construction); contrairement aux autres quartiers, les chefs de famille n'ont pas de parents agriculteurs, et ils sont principalement nés à La Paz. Les caractéristiques démographiques des familles (nombre élevé d'enfants, couples entre 40 et 60 ans) laissent penser que ces quartiers, créés au tournant des années 1990-2000, accueillent des familles désireuses d'accéder à la propriété, mais qui ne peuvent assumer un coût de l'immobilier désormais trop élevé dans des zones plus centrales de la ville. Ces quartiers sont aussi les seuls à comporter le moins de maisons d'une seule pièce en adobe (torchis), et le plus de maisons en briques de plusieurs étages, avec cuisine séparée, toilettes, et sol en ciment, se rapprochant ainsi des standards de confort du centre-ville où les chefs de famille se rendent majoritairement dans leur véhicule propre. Même si leurs moyens restent modestes, les familles du pôle en voie d'équipement ont suffisamment de ressources économiques pour pouvoir surmonter les obstacles à l'installation individuelle de services de base. Ce sont aussi celles qui affirment le plus fortement la demande de connexion au réseau d'eau potable. Un capital économique suffisant permet donc de surmonter les difficultés techniques liées à la localisation du quartier, mais dans les quartiers périphériques en expansion, cette situation reste minoritaire. Si le niveau de vie des populations résidentes (en relation avec leurs origines sociales et les professions exercées) permet d'opposer, sur l'axe 1, le pôle constitué par les quartiers en voie d'équipement à tous les autres, il ne suffit cependant pas à expliquer la disparité des situations. Les autres quartiers ne sont pas "équipables», au moins à court terme, mais pour des raisons différentes qui ne sont pas seulement économiques. Les plus en difficulté, comme Pokechaka (en bas à gauche), cumulent d'une part les obstacles techniques liées à une situation de surplomb par rapport au réseau, 


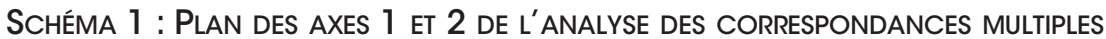 \\ Quartiers périphériques en extension de La Paz Caractéristiques socioéconomiques d'accès à l'eau}

QUARTIERS NON ÉQUIPÉS (RAISONS TECHNIQUES)

1 ou 2 pièces

Brique

2 ou 3 enfants jeune couple

Installation dans les années 1990

ALTO OVEJUYO

Investissement supplémentaire trop cher

Puits dans le quartier

Accessibilité gestion informelle

intégration socioéconomique +

Légende
QUARTIERS
Type de logement
Caractéristiques du chef de famille
Perception du service et disposition à s'équiper
Accès à l'eau

l'eau

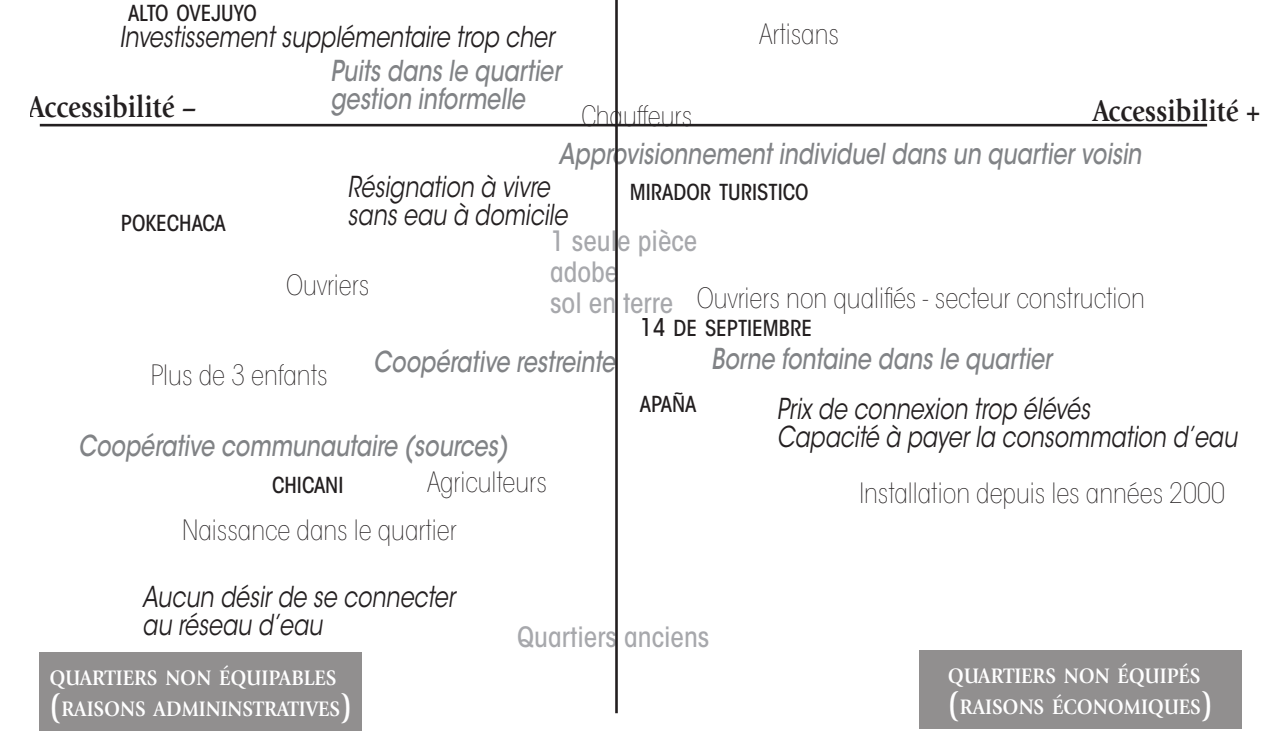

intégration socioéconomique - 
et une situation de précarité des chefs de famille résidents (ouvriers non qualifiés et non salariés, journaliers, retraités). Ces problèmes ont des conséquences administratives directes: absence d'autorisation municipale concernant la voirie dans des zones trop instables, non-obtention de titres de propriété due à la proximité de décharge toxique, etc. Dans ce pôle non équipable, Chicani présente une situation quelque peu particulière: cette zone, où réside la plus forte proportion d'exploitants agricoles (30\% de l'échantillon territorial) tirant de modestes revenus de petites parcelles individuelles, possède plusieurs coopératives qui exploitent des eaux de source abondantes et qui desservent les habitations avec un système de canalisations à domicile. Cette spécificité de Chicani explique qu'en dehors de la présence des agriculteurs, ce quartier soit le plus proche socialement du pôle en voie d'équipement, avec un pourcentage de professions intermédiaires et indépendantes (construction, transports) supérieur à la moyenne de l'échantillon, et des habitations de plusieurs étages ou de plusieurs pièces, construites en briques, etc.

Entre ces deux pôles opposés se trouvent tout d'abord les quartiers (en bas à droite) qui ne sont pas équipés pour des raisons économiques (Mirador Turístico, Apaña, 14 de Septiembre) : en l'absence de difficultés majeures en termes d'accessibilité matérielle, c'est la faible capacité de paiement individuelle des habitants, souvent installés depuis peu dans ces zones périphériques en extension et trop peu denses, qui constitue l'obstacle principal à court terme. La croissance de la population des quartiers laisse cependant présager des possibilités d'équipement à plus long terme; l'accessibilité du quartier rendra possible une connexion au réseau dès lors que les conditions collectives seront réunies : reconnaissance de la planimétrie auprès de la mairie, un seuil de rentabilité fixé à $50 \%$ d'occupation des lots requis par l'entreprise municipale pour commencer les travaux, création et mobilisation d'un comité de quartier pour réaliser le suivi des démarches. Si les difficultés des familles prises individuellement sont avant tout économiques, c'est cependant l'axe 2 exprimant le niveau d'accessibilité des quartiers qui permet de comprendre les obstacles à l'équipement collectif en services urbains. Les quartiers non équipés pour raisons économiques s'opposent ainsi aux zones (en haut à gauche) dont l'accès au service de distribution d'eau se heurte à des difficultés d'ordre technique, principalement liées à leur localisation (Alto Ovejuyo, 24 de Junio) : situées dans des conditions géographiques difficiles, sur les pentes surélevées des montages environnantes, ou séparées du centre urbain par un obstacle naturel (roches, cours d'eau, etc.), ces dernières nécessitent l'installation d'un réseau spécial, destiné à contourner les problèmes de pression, ce qui augmente significativement les coûts d'installation pour les habitants. Ces zones possèdent la plus forte proportion de chefs de famille de moins de 40 ans : une partie non négligeable des résidents, lorsqu'ils n'ont pas toujours vécu dans le quartier, y viennent pour accéder à la propriété et obtenir plus d'espace pour leurs enfants. Sur l'ensemble de l'échantillon, seuls les quartiers de Pokechaka et 24 de Junio semblent correspondre à un modèle de migration traditionnel, du monde rural vers la ville : ils accueillent un pourcentage élevé de gens étant nés hors du département de La Paz, et une fraction non négligeable d'entre eux parlent quechua (et non aymara), signe d'une naissance sur des parties éloignées de l'altiplano (Oruro, Potosi, voire dans les vallées descendant sur Cochabamba). Par ailleurs, les quartiers d'implantation la plus ancienne, comme Chicani, Apaña 
SCHÉMA 2 : ReprésentATION SIMPLIFIÉE DES INDICATEURS SOCIOSPATIAUX D'ACCÈs À L'EAU

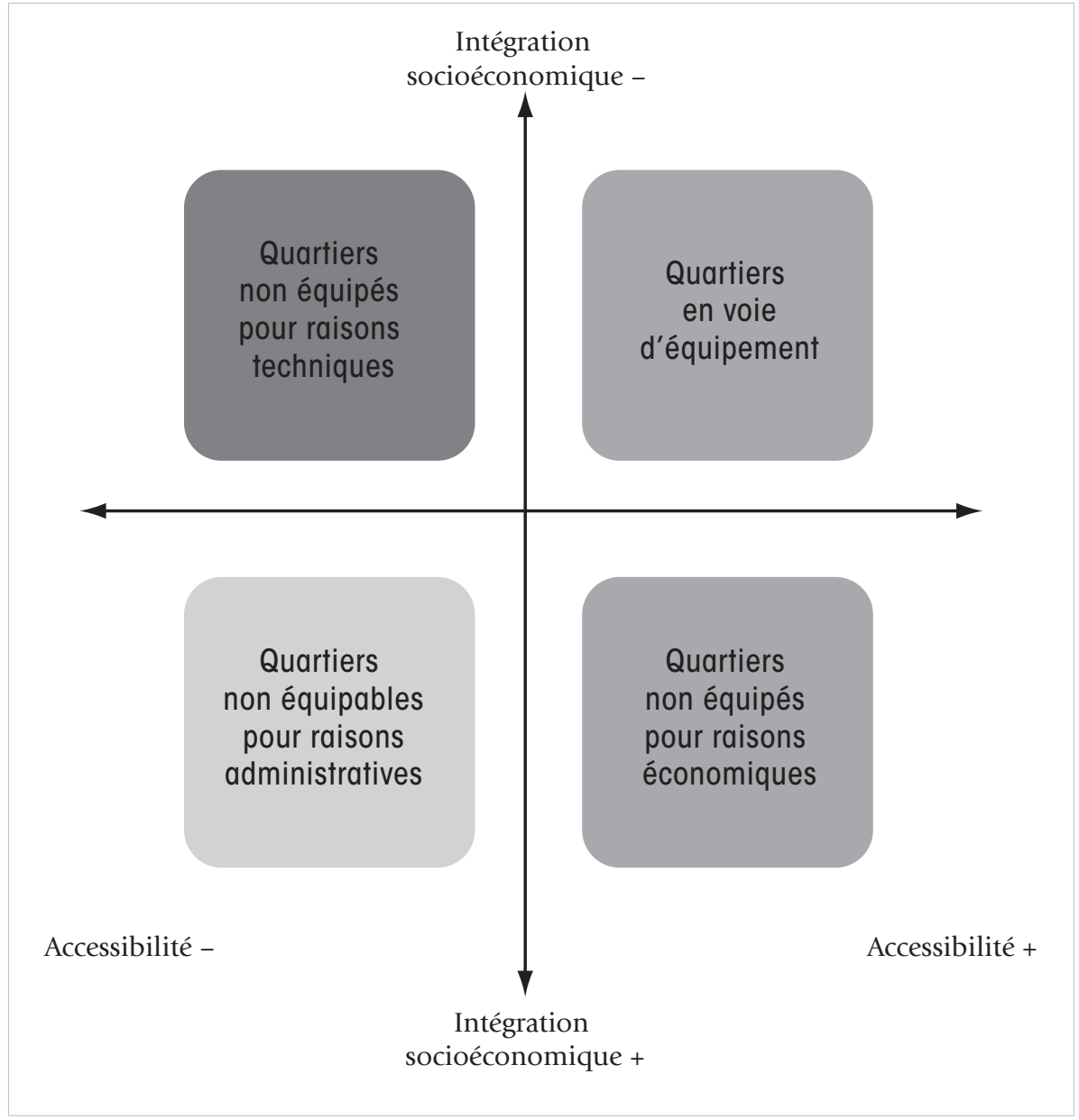

et Alto Ovejuyo, sont aussi ceux qui comportent le plus de chefs de famille de sexe masculin, et dont les parents résident dans la même maison, indiquant la persistance d'un mode de reproduction économique à dominante familiale. Du point de vue des conditions de logement, ces quartiers (tout comme les non-équipables) se caractérisent par un habitat particulièrement sommaire, si l'on s'en tient à des indicateurs comme le sol en terre ou l'existence d'une pièce commune unique. Certains comptent cependant de plus en plus de murs en briques, comme Mirador Turístico : situé dans une zone rocheuse où il est difficile de fabriquer de l'adobe (les résidents des zones d'autoconstruction utilisent la terre creusée sur place pour construire leurs logements), c'est aussi un quartier très récent, où la plupart des habitants sont installés depuis 2002. L'ancienneté de l'installation se répercute sur les anticipations des résidents : ceux qui pensent agrandir leur maison et qui sont le plus en demande d'équipement urbain sont ceux qui sont dans les quartiers les plus récents, ou qui possèdent les foyers implantés le plus récemment. 
On voit donc qu'il n'y a pas d'échelle unique pour classer les quartiers d'un point de vue sociodémographique: une vraie diversité préside aux modes d'installation, qu'il s'agisse de l'ancienneté, de la composition familiale ou du rapport au quartier. Ces variables constituent en revanche des ensembles cohérents (les pôles pré-mentionnés) dès qu'elles sont ordonnées à partir des conditions sociospatiales d'accès au service d'eau. Les inégalités entre les quartiers s'expriment ainsi dans la distance entre le domicile et le lieu de travail, et le coût des transports qui en résulte. Pour les quartiers en voie d'équipement, la présence d'automobiles et de transports directs avec le centre urbain explique les faibles dépenses. Dans les autres quartiers en revanche, les dépenses de transport représentent une part conséquente du budget, avec plusieurs changements de lignes de bus pour les employés travaillant en centre-ville. Seules quelques zones impliquent un temps de transport inférieur à 30 minutes par jour : Chicani, où des parcelles sont cultivées par les agriculteurs locaux; les quartiers non équipés pour raisons techniques d'Alto Ovejuyo et 24 de Junio, dont la proximité avec le centre-ville se paie par des conditions d'accès plus difficiles, en surplomb des axes de transports principaux. On voit donc l'imbrication des contraintes spatiales et des caractéristiques sociales : l'espace n'est pas une donnée "en soi», et les obstacles ou les facilités qu'il procure dépendent autant des caractéristiques des familles résidentes que des ressources qu'elles peuvent mobiliser afin de s'affranchir des contraintes spatiales. Inversement, on ne peut établir de correspondance directe entre la profession exercée et le type de quartier habité : on ne va pas forcément dans un quartier plus périphérique et plus lointain parce qu'on a un travail moins bien payé. La localisation apparaît bien plutôt comme le produit de différentes contraintes, dont la relation au lieu de travail constitue une dimension parmi d'autres.

\section{Styles de vie et prises de positions politiques}

L'analyse des quartiers à partir de l'habitat et des caractéristiques démographiques, sociales et économiques des familles permet de mettre en évidence la cohérence des styles de vie (habitudes hygiéniques, dépenses, etc.) et des prises de position politiques des habitants par rapport au service de distribution des eaux. Comme on pouvait s'y attendre, Chijipata, le quartier le plus aisé, abrite le plus grand nombre de résidents se douchant chaque jour. Dans les autres quartiers, les adultes qui n'ont pas de douche à domicile le font principalement au travail, sauf à Chicani où la présence de sources abondantes permet une hygiène corporelle plus régulière. Le nombre de toilettes à domicile fait apparaître des disparités importantes : le coût des installations, qu'il s'agisse d'une évacuation vers une fosse d'aisances (pozo ciego) ou de la capacité de réserver une pièce dans le domicile pour les toilettes, s'avère prohibitif pour les ménages les plus démunis. Les habitudes hygiéniques des familles se trouvent donc au croisement des deux dimensions sociales et spatiales mises en évidence précédemment : le manque de moyens économiques accentue la dépendance envers les ressources naturelles locales. Du point de vue des usages quotidiens, le questionnaire fait apparaître un recours prédominant aux eaux de pluie pour cuisiner, aux eaux de source et aux puits pour laver le linge et la vaisselle. Il existe aussi des approvisionnements alternatifs : achats de l'eau aux voisins (quartiers non équipés pour raisons économiques), utilisation d'une borne fontaine 
aux frontières du quartier (quartiers non équipés pour raisons techniques). On comprend alors pourquoi les conflits qui affectent ces zones périphériques frontalières ont toujours un rapport, direct ou indirect, avec les problèmes d'accès à l'eau : soit parce qu'il y a dépendance envers les zones desservies, soit parce que les résidents des zones desservies réclament d'être rattachés à La Paz et non aux municipalités voisines pour bénéficier du service.

Les questions relatives aux motivations pour se connecter au service d'eau et d'assainissement font apparaître d'autres lignes de clivage. Les raisons de sécurité (ne pas avoir à sortir la nuit pour se rendre aux toilettes) sont invoquées majoritairement dans tous les quartiers, tandis que le fait de ne pas avoir de conflits avec les voisins apparaît important dans les quartiers où est installée une coopérative (Chicani). Les antagonismes entre les dirigeants des coopératives et les résidents qui en sont exclus sont récurrents: ces derniers réclament une connexion au réseau que les bénéficiaires des coopératives refusent par crainte des coûts supplémentaires. À la question de savoir s'ils seraient prêts à investir de l'argent pour accéder au réseau d'eau potable, les habitants de tous les quartiers répondent principalement par l'affirmative, de même qu'ils estiment majoritairement que payer une facture pour consommer l'eau est normal - à l'exception, là encore, des usagers des coopératives rurales qui, bénéficiant d'un accès abondant et gratuit à la ressource, ne veulent pas changer de mode de gestion. Mais lorsqu'on leur demande quand ils pensent faire la connexion, les habitants des différents quartiers ne manifestent pas le même souci pour s'équiper: seuls les quartiers non équipés pour raisons techniques ont véritablement entrepris des démarches; dans les autres cas, les résidents attendent de l'entreprise de distribution qu'elle engage les travaux d'installation du réseau. Même si la demande de connexion s'exprime majoritairement sous la forme de raccordement au domicile individuel, l'accès au service reste perçu comme le résultat d'un processus collectif : plus de $80 \%$ des réponses dans tous les quartiers font apparaître que c'est parce que la zone n'est pas connectée que l'accès individuel n'est pas réalisé. Du point de vue des prix justement, la plupart des résidents des quartiers enquêtés avouent ignorer le tarif de la connexion individuelle au réseau. Une fois les prix connus, leurs velléités de connexion chutent sensiblement, mais de façon différenciée selon les situations et les perceptions du service de distribution. C'est à ce niveau que les styles de vie expriment des inégalités sociospatiales plus larges.

Si, comme on l'a déjà noté, la demande de services urbains est la plus forte dans les quartiers en voie d'équipement, les attitudes sont contrastées dans les autres quartiers : la forte demande des quartiers non équipés pour raisons techniques va de pair avec le refus d'investir plus que nécessaire afin de compenser les obstacles au raccordement au réseau; les quartiers non équipés pour raisons économiques, tout comme ceux qui ne sont pas équipables, laissent apparaître des formes de résignation à vivre sans eau à domicile. Les entretiens menés avec les habitants montrent cependant une faible politisation des résidents par rapport à la situation qu'ils vivent. Plusieurs indicateurs le confirment: tout d'abord, l'évaluation des tarifs de la consommation d'eau entraîne un relatif consensus sur l'obligation de payer pour bénéficier d'un service de distribution régulier et de qualité : la revendication 
d'un «droit à l'eau» promue par les organisations non gouvernementales, nationales ou internationales, ne trouve encore aucun écho dans la population. Seul Chicani se distingue par une plus faible proportion de gens disposés à payer, dans la mesure où l'abondance de l'eau acheminée par la coopérative depuis les sources rend inutile la tarification du service. Mais plus généralement, ce sont surtout les quartiers où la ressource n'est pas disponible qui possèdent le plus fort taux de résidents sans opinion politique. On a pu constater en effet qu'une grande majorité des personnes interrogées n'avaient pas connaissance de la remunicipalisation du service de distribution depuis 2007, sauf à Alto Ovejuyo, où des démarches avaient été entreprises quelques années auparavant par une décision commune des résidents. Considérés comme indicateurs de prise de position politique, les avis rendus (positifs ou négatifs) sur le départ de l'entreprise privée sont les plus faibles dans les quartiers où les enquêtés se sentent davantage rattachés à un district rural en marge de la ville qu'à une zone urbaine desservie par l'entreprise, ainsi que dans ceux qui ont peu d'espoir de se voir équipés à court terme, malgré des demandes répétées auprès de l'administration, en raison des problèmes techniques rencontrés; inversement, les taux de sans opinion sur le changement d'entreprise diminuent sensiblement dans les quartiers dotés d'une qualité de vie ou d'un niveau scolaire supérieurs (Chijipata, les condominiums et dans une moindre mesure 24 de Junio). Ces résultats sont cependant à manier avec prudence: le taux de non-réponse indique chez les habitants à la fois un fort sentiment de dépossession engendrant des préoccupations éloignées des thématiques politiques "officielles » et de l'actualité, et une incertitude supplémentaire dans le recueil d'informations liée au fait que les personnes interrogées ne se sentent pas autorisées à exprimer "publiquement» (i.e., auprès des enquêteurs étrangers) leurs opinions politiques, selon la logique classique des relations entre capital scolaire et capital politique ${ }^{16}$. Ces incertitudes des réponses se retrouvent dans la perception des problèmes de rareté et de contamination de l'eau : les opinions émises dépendent de la situation particulière du quartier plus que d'un avis informé sur les problèmes environnementaux. Et excepté dans les quartiers disposant de sources propres alimentant une coopérative, on peut constater que seule une minorité des résidents déclarent bénéficier d'une eau de bonne qualité; ainsi Alto Ovejuyo, dont l'unique puits s'avère peu avenant pour la consommation, et les quartiers où les sources ne sont pas très abondantes ( 24 de Junio et Pokechaka), expriment des taux de satisfaction particulièrement faibles envers la qualité de l'eau.

\section{Conclusion : indicateurs d'inégalités et espace social}

Les difficultés que les quartiers périphériques rencontrent pour accéder à l'eau livrent plusieurs enseignements sur le processus de métropolisation. Les conflits générés par le flou des frontières entre les municipalités renvoient tout d'abord aux caractéristiques spécifiques des différentes populations résidentes. Ces conflits ne relèvent pas de simples divergences d'intérêts économiques (payer plus ou

16 - P. Bourdieu, La Distinction. Critique sociale du jugement, Paris, Minuit, 1979. 
moins d'impôts locaux), mais tiennent à des différences de styles de vie et d'aspirations - aspirations urbaines pour une grande partie des résidents des zones frontalières - qui sont modulées par la distance et les obstacles jusqu'aux parties centrales de la ville. L'analyse des inégalités à partir de l'indicateur d'accès à l'eau montre l'importance des dimensions indissociablement spatiales et sociales des formes de ségrégation urbaine. La cohérence des styles de vie associés au fait de vivre dans tel ou tel quartier périphérique apparaît dans l'interaction des variables spatiales, exprimées dans la configuration de chaque quartier, et des variables sociales, résumées dans les caractéristiques des chefs de famille. L'enquête permet alors d'établir les principes d'opposition selon lesquels se structure l'espace social de la métropole bolivienne: résidence dans les quartiers périphériques récents/ dans des quartiers anciens, grande/faible distance avec le lieu de travail, propriétaires/locataires, habitat non équipé/habitat équipé en services urbains, maison en adobe/maison en briques, hygiène ponctuelle/hygiène quotidienne, consommation alimentaire basée sur les pommes de terre/alimentation variée, ouvriers du bâtiment/commerçant(e)s, travail précaire/petite entreprise familiale, études primaires/études secondaires, aspirations urbaines/liens actifs avec le monde rural, mobilisation politique faible/revendications identitaires, etc.

À travers les inégalités constitutives de l'espace urbain se révèlent ainsi les structures d'un espace social fortement segmenté, qui façonnent l'ensemble des styles de vie, depuis le matériau du sol de la maison jusqu'aux prises de position politiques. Une des principales conséquences de ces résultats est que, dans le cas des périphéries en expansion des pays du Sud comme celles de La Paz, il n'est pas possible de raisonner sur une échelle unique de "pauvreté», mais il faut construire des indicateurs sociospatiaux intégrant plusieurs dimensions. Être ouvrier du bâtiment ou employé n'a pas la même signification selon qu'on habite dans un quartier accessible ou non; être en bout de réseau, confronté à des obstacles naturels élevant les coûts d'installation, devoir dépenser plus en transports, etc., constituent autant de facteurs d'appauvrissement relatif, et rendent encore plus problématique l'accès à des services urbains de base, qui ont en retour des effets importants sur les styles de vie familiaux. Dans cette perspective, les indicateurs sociospatiaux permettent de compléter l'information fournie par les indicateurs classiques comme la profession ou le revenu: l'étude des logiques constitutives de l'espace urbain offre ainsi de nouvelles pistes de recherche pour comprendre les transformations des structures de l'espace social et des inégalités qui le traversent. 


\section{Bibliographie}

- Agrer M., L'invention de la ville, Amsterdam, Overseas Publishers Association et Paris, Éditions des archives contemporaines, 1999.

- Aguilar A. G. et P.M. WArd, «Globalization, regional development and mega-city expansion in Latin America: analyzing Mexico City's peri-urban hinterland", Cities, 20 (1), 2003, p.3-21.

- Albó X., T. Greaves et G. SAndoval, Chukiyawu, la cara aymara de La Paz, t. IV, Nuevos Lazos con el campo, La Paz, CIPCA, 1987.

- Bairoch P., Le Tiers Monde dans l'impasse: le démarrage économique du XVIII ${ }^{e}$ au $\mathrm{XX}^{e}$ siècle, Paris, Gallimard, 1992 [1971].

- Bourdieu P., La Distinction. Critique sociale du jugement, Paris, Minuit, 1979.

- Gobierno Municipal de La Paz, Atlas del municipio de La Paz. Una lectura sociodemográfica desde las Organizaciones Territoriales de Base, La Paz, CODEPO - GMLP - IRD, 2006.

- Komives K., «Designing pro-poor water and sewer concessions : early lessons from Bolivia», Water Policy, 2001 (3), p. 61-79.

- Lacour C. et S. Puissant, la métropolisation. Croissance, diversité, fractures, Paris, Anthropos, 1999.

- Lebaron F., L'enquête quantitative en sciences sociales. Recueil et analyse des données, Paris, Dunod, 2006.
- Meublat G., «La rénovation des politiques de l'eau dans les pays du Sud», Revue Tiers Monde, t. XLII, n 166, 2001, p. 249-258.

- Poupeau F., «Las desigualdades de acceso al agua en El Alto", contribution au séminaire international sur "Modelos de gestión del agua en ciudades y comunidades de los Andes", IFEA - PIEB, La Paz, 58 novembre 2007 .

- Poupeau F., «Les ambivalences de la participation communautaire. Le cas du service de distribution des eaux à El Alto, Bolivie», Autrepart, n 47, 2008, p. 245-254.

- Poupeau F., «De la migración rural a la movilidad intra-urbana. Una perspectiva sociológica sobre las desigualdades socioespaciales de acceso al agua en El Alto, Bolivia», in F. Poupeau et C. Gonzales (dir.), Modelos de gestión del agua en los Andes, IFEA - PIEB, 2009.

- Poupeau F., «El Alto: una ficción política», conférence prononcée à l'Université de tous les savoirs, des Andes à l'Amazonie, le 21 septembre 2009 à La Paz, Bolivie.

- Pumain D. et T. Saint-Julien, 2001, Les interactions spatiales: flux et changements dans l'espace géographique, Paris, Armand Colin, 2001.

- Troin J.-F., Les métropoles des Sud, Paris, Ellipses, 2000. 